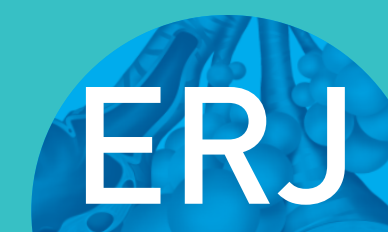

open research
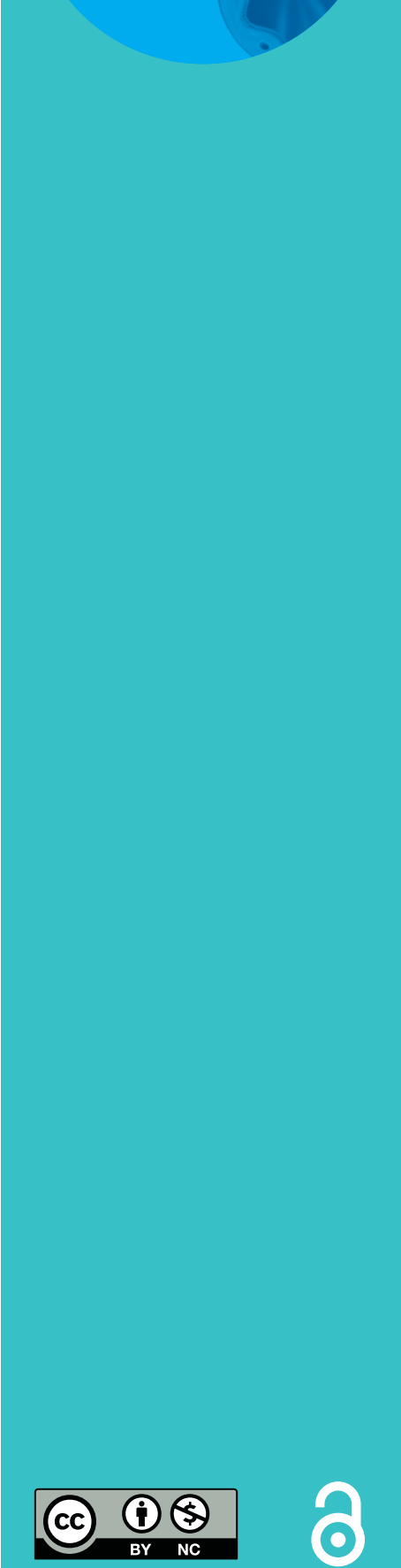

\section{Sleep clinical record: what differences in school and preschool children?}

\author{
Maria Pia Villa ${ }^{1}$, Hanaa Shafiek ${ }^{2}$, Melania Evangelisti ${ }^{1}$, Jole Rabasco ${ }^{1}$, \\ Manuela Cecili ${ }^{1}$, Marilisa Montesano ${ }^{1}$ and Mario Barreto ${ }^{1}$
}

Affiliations: ${ }^{1}$ Pediatric Sleep Disease Center, Child Neurology, NESMOS Dept, School of Medicine and Psychology, Sapienza University of Rome, S. Andrea Hospital, Rome, Italy. ${ }^{2}$ Dept of Chest Diseases, Faculty of Medicine, Alexandria University, Alexandria, Egypt.

Correspondence: Maria Pia Villa, Pediatric Sleep Disease Center, Child Neurology, NESMOS Dept, School of Medicine and Psychology, Sapienza University of Rome, S. Andrea Hospital, Via di Grottarossa 1035-39, 00189 Rome, Italy. E-mail: mariapia.villađuniroma1.it

ABSTRACT The sleep clinical record (SCR) may be a valid method for detecting children with obstructive sleep apnoea (OSA). This study aimed to evaluate whether there were differences in SCR depending on age and to identify the possible risk factors for OSA development.

We enrolled children with sleep disordered breathing between 2013 and 2015, and divided them according to age into preschool- and school-age groups. All patients underwent SCR and polysomnography.

OSA was detected in $81.1 \%$ and $83.6 \%$ of preschool- and school-age groups, respectively. Obesity, malocclusions, nasal septal deviation and inferior turbinate hypertrophy were significantly more prevalent in school-age children $(\mathrm{p}<0.05)$; however, only tonsillar hypertrophy had significant hazard ratio (2.3) for OSA development. Saddle nose, nasal hypotonia, oral breathing and tonsillar hypertrophy were significantly more prevalent for development of OSA in preschoolers $(p<0.03)$. The SCR score was significantly higher among preschool children than in school-age children $(8.4 \pm 2.22$ versus $7.9 \pm 2.6$; $\mathrm{p}=0.044$ ). Further, SCR score $>6.5$ had a sensitivity of $74 \%$ in predicting OSA in preschool children with positive predictive value of $86 \%(\mathrm{p}=0.0001)$.

Our study confirms the validity of the SCR as a screening tool for patient candidates for a PSG study for suspected OSA, in both school and preschool children.

@ERSpublications

The differences in sleep clinical record between school and preschool children suspected to have OSA http://ow.ly/X778Q

Received: Aug 032015 | Accepted after revision: Dec 292015

Conflict of interest: None declared.

Copyright $\odot$ ERS 2016. This article is open access and distributed under the terms of the Creative Commons Attribution Non-Commercial Licence 4.0. 


\section{Introduction}

Obstructive sleep apnoea syndrome (OSAS) is characterised by repeated episodes of prolonged partial upper airway obstruction (obstructive hypopnoea) and/or intermittent complete obstruction (obstructive apnoea) that disturb normal sleep pattern and normal ventilation during sleep, resulting in disruption of normal gas exchange (intermittent hypoxia and hypercapnia) [1-3].

The prevalence of OSAS has been traditionally estimated to be $1-5.7 \%$ in the paediatric population [4-6]. In 2011, the American Academy of Sleep Medicine (AASM) published the practice parameters for the respiratory indications for polysomnography (PSG) in children with sleep disordered breathing (SDB) [7]. It has been reported that evaluation for SDB mostly based on clinical evaluation alone does not have sufficient specificity and sensitivity to establish a diagnosis of OSAS and the gold standard for the diagnosis of SDB is overnight PSG; however, it is also an expensive, time consuming and labour intensive procedure [8].

Several questionnaires have been created and developed in an effort to find a simple screening instrument to identify paediatric subjects who are at high risk for OSAS. Various aetiological factors may underlie OSAS in the paediatric population, including obesity, various craniofacial disorders and hypotonic neuromuscular diseases. The most common cause is thought to be adenotonsillar hypertrophy. It is important to distinguish children with different levels of severity of OSAS using the patient's clinical history and physical examination to identify children in need of PSG prior to adenotonsillectomy. The sleep clinical record (SCR) is a rapid, validated instrument that may effective when used to screen patient candidates for a PSG study for suspected OSAS [8].

Accordingly, the aims of our study were to: evaluate the SCR as a screening tool for suspected OSAS in preschool children; identify whether there were differences in SCR parameters either related to physical examination (including nose, oropharynx, dental and skeletal occlusion) or presenting symptoms according to age; and to identify the possible risk factors for OSAS development at different ages.

\section{Methods}

\section{Study design and population}

We enrolled 420 children who attended the Pediatric Sleep Disease Centre (Rome, Italy) between 2013 and 2015. Children with genetic disorders, cerebral palsy, neuromuscular diseases, major craniofacial abnormalities, associated chromosomal syndromes and seizures were excluded from the current study. The children were divided into two groups according to their age: preschool children ( $<6$ years old; 292 children, 69.5\%) and school-age children ( $\geqslant 6$ years old; 128 children, 30.5\%). All patients underwent laboratory PSG, where the diagnosis of OSAS was confirmed in cases with an obstructive apnoea-hypopnoea index $(\mathrm{AHI}) \geqslant 1 \mathrm{event}^{-1} \mathrm{~h}^{-1}$ according to AASM criteria [7, 9]. Accordingly, the preschool group was divided into two subgroups according to AHI: the obstructive sleep apnoea (OSA) subgroup and habitual snoring subgroup, i.e. an AHI $<1$ event $\mathrm{h}^{-1}$ and microphone-detected snoring, for further evaluation. The study protocol was approved by the local Ethics Committee and written informed consent was obtained from the parents.

\section{Characteristics of the participants}

All children underwent a full clinical history through a questionnaire completed by their parents (including the child's age; age of symptoms onset; sleep symptoms such as habitual snoring, witnessed apnoeic episodes and frequent awakenings or restless sleep). The weight (in $\mathrm{kg}$ ) and height (in $\mathrm{cm}$ ) were taken at presentation. The body mass index (BMI) was calculated as well as the BMI percentile (pBMI). Children with $\mathrm{pBMI} \geqslant 95$ th percentile were considered obese according to the International Obesity Task Force. All children underwent a general clinical examination and an ear, nose, and throat (ENT) and orthodontic assessment.

\section{Sleep clinical record}

All the children underwent SCR evaluation, as previously validated by VILLA et al. [8]. Briefly, SCR consists of three main items. First, the data yielded by physical examination of the nose, oropharynx and dental and skeletal occlusion. The following signs were considered: signs of oral breathing such as nasal cartilage hypotonia, orbicular muscle hypotonia or rhinolalia; nasal obstruction, considering nostril patency (analysed by blocking one nostril with one finger followed by inspiration through the non-occluded nostril) and inferior turbinate hypertrophy; dental/skeletal malocclusion including pathological palate position graded according to Friedman classes (grade 3 and 4 considered positive) [10]; nasal septum deviation; tonsillar hypertrophy (grades 3 and 4) [11]; obese or adenoid phenotype; and narrow palate. These signs were scored as 2 points (positive sign) versus 0 points (negative sign). Secondly, Brouillette score was calculated from the presented symptoms, mainly apnoea, snoring and restless sleep, and scored 
as 0.5 points provided that the calculated score was $\geqslant-1$ [12]. Thirdly, the presence of symptoms of inattention and hyperactivity using the attention deficit hyperactivity disorder (ADHD) rating scale for school-aged children, adapted Italian version [13], was considered. An ADHD scale of more than 6 was considered positive and scored as 1 point. A total SCR score of 6.5 was considered positive [8].

\section{PSG and sleep stage scoring}

All children underwent laboratory overnight PSG assessment using a Grass Heritage polygraph (Natus Neurology Incorporated - Grass products, Warwich, RI, USA). The variables recorded included an electroencephalogram (EEG) with at least six channels (bilateral frontal, central, temporal and occipital monopolar montages referred to the contralateral mastoid), an electrooculogram (electrodes placed $1 \mathrm{~cm}$ above the right outer cantus and $1 \mathrm{~cm}$ below the left outer cantus and referred to A1), submental electromyogram (EMG) and ECG (1 derivation). Sleep stages were scored according to the standard criteria of the AASM [9] after subdividing into 30-s epochs. The percentage of total sleep time in each stage was measured as follows: percentage of stage $\mathrm{N} 1$, stage $\mathrm{N} 2$, stage $\mathrm{N} 3$ and stage R (rapid eye movement sleep). Arousals were detected visually according to the criteria reported in the AASM manual for the scoring of sleep and associated events [9].

Central, obstructive and mixed apnoea events were counted according to the criteria established by the AASM [9]. Chest and abdomen movements were measured by strain gauges. Oronasal airflow was recorded by means of a thermocouple and nasal pressure. Arterial oxygen saturation was monitored using pulse oximetry. The obstructive AHI was defined as the average number of obstructive apnoeas and hypopnoeas per hour of sleep. The diagnosis of OSAS was confirmed by means of a laboratory PSG revealing an obstructive AHI $\geqslant 1$ event $\cdot h^{-1}$ [9]. Primary snoring was diagnosed in children with habitual snoring, an AHI $<1$ event. $h^{-1}$ and microphone-detected snoring.

\section{Statistical analysis}

Values are expressed as mean \pm SD for continuous variables and number and percentage for categorical variables. All variables were tested for normality. Chi-squared test or Fisher's exact test has been used for categorical variables and independent t-test or Mann-Witney test for continuous variables according to the normal distribution of the available data. Spearman's rho correlation was used to investigate the variables of interest. Receiver operating characteristic (ROC) curve and area under the curve (AUC) were used to calculate the sensitivity, specificity, positive predictive value (PPV) and negative predictive value (NPV) of the SCR for predicting OSA in preschool children. Power analysis for evaluating the SCR was calculated for our sample size considering $\alpha$ (type I error) equal to 0.05 . Univariate and multivariate logistic regression hazard analyses were applied to study different parameters of the SCR in relation to age. Hazard ratios and 95\% confidence intervals are reported. All the conducted tests were two-tailed and $\mathrm{p}<0.05$ was considered significant. An SPSS package (PASW Statistics for Windows, Version 18.0. Chicago: SPSS Inc. 2009) was used for the analysis.

\section{Results}

\section{Study population}

The clinical characteristics of the recruited children are summarised in table 1 . The mean $\pm \mathrm{SD}$ age of preschool children was 3.96 \pm 1.2 years (range $0.91-5.91$ years) and that of school-age children was $9.1 \pm 2.4$ years (range $6.0-17.16$ years). In both groups male sex was more frequent $(65 \%)$. Obesity was significantly more prevalent among the school-age group than among the preschool group (40.6\% versus $14.5 \%$; $\mathrm{p} 0.0001)$. Furthermore, the SCR score was significantly higher in preschool children compared with the school-age group $(8.4 \pm 2.22$ versus $7.9 \pm 2.6 ; \mathrm{p}=0.044)$. OSA was detected in $81.1 \%$ of the preschool group, with a mean \pm SD AHI of $8.2 \pm 8.9$ events $\cdot \mathrm{h}^{-1}$, and $83.6 \%$ of school-age group, with a mean \pm SD AHI of $7.7 \pm 10.5$ events $\cdot h^{-1}(p>0.05$; table 1$)$. Variable age of onset and duration of symptom development were detected in the children studied.

\section{SCR and PSG}

Malocclusions (retrognathia/prognathia) and overjet were significantly more common among school-age children ( $35.2 \%$ (45 children) and $11.7 \%$ (15 children); $\mathrm{p}=0.022$ and 0.001 , respectively) (table 1 ). The majority of children with angle malocclusion (40 out of 45 children) were proved to have OSA by PSG as well as for overjet (12 out of 15 children). Furthermore, nasal septal deviation, mucosal hyperaemia and inferior turbinate hypertrophy were significantly more prevalent among school-age children $(\mathrm{p}<0.05$; table 1$)$. However, nasal hypotonia, habitual nasal obstruction, saddle nose, oral breathing, high arched palate, open bite and tonsillar hypertrophy were significantly more common among the preschool group $(\mathrm{p}<0.05$; table 1). In addition, apnoea was a significantly more frequent presentation among the preschool group $(\mathrm{p}<0.0001 ;$ table 1$)$. 


\section{TABLE 1 Demographic and clinical differences between preschool and school-age children}

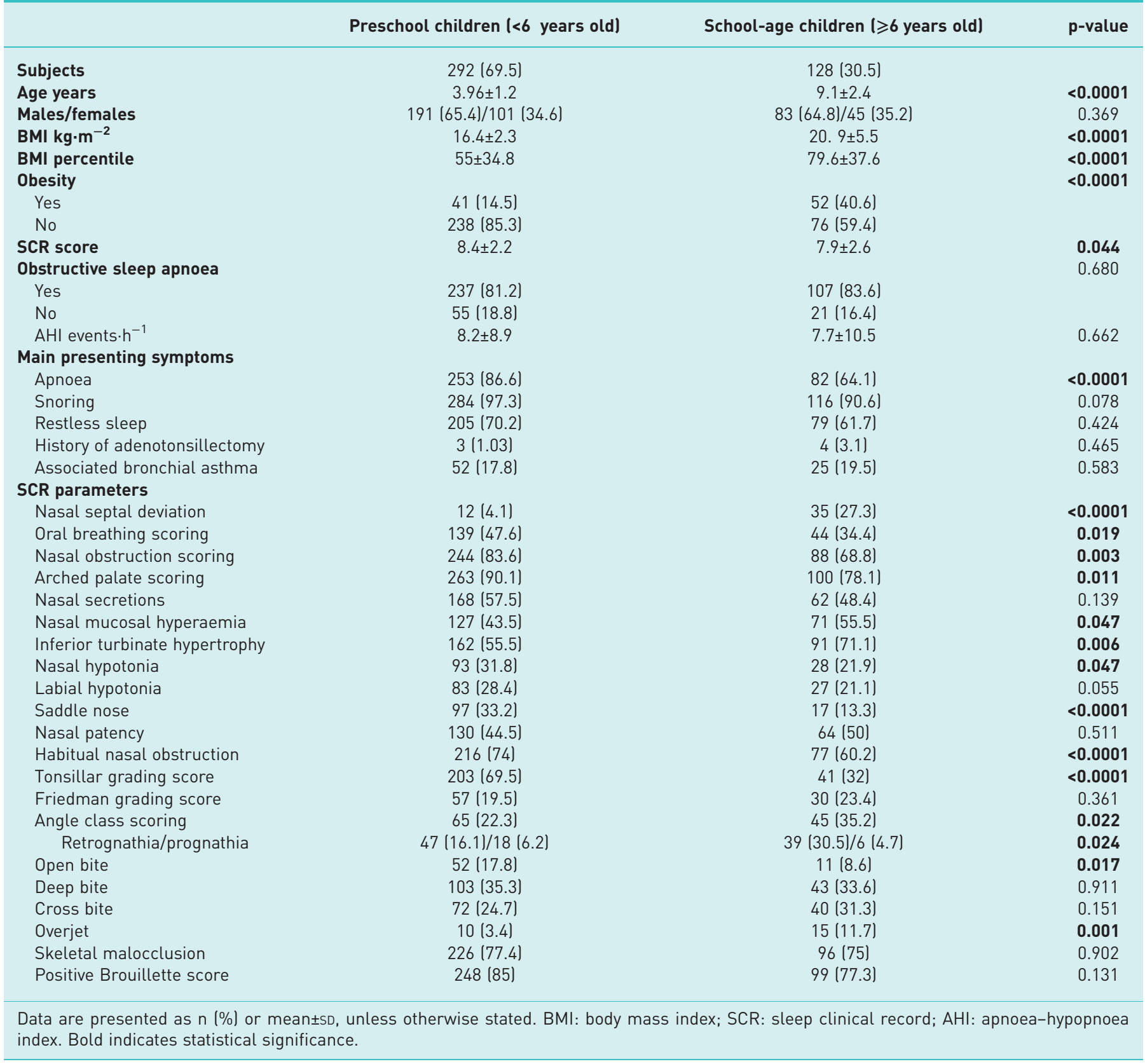

$81 \%$ (237 children) of preschool children were shown to have OSA with mean \pm SD AHI of $8.2 \pm 8.9$ events $\cdot \mathrm{h}^{-1}$,

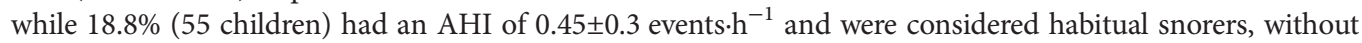
a significant difference regarding the age of onset $(\mathrm{p}=0.308)$. We analysed the SCR using the ROC curve, which showed a score $>6.5$, providing that ADHD is not included for this age group, had a sensitivity of $74 \%$ in predicting OSA in preschool children (AUC 0.663, 95\% CI 0.606-0.717; $\mathrm{p}=0.0001$ ) (figure 1), with a PPV of $86 \%$ and positive and negative likelihood ratio of 1.5 and 0.53 , respectively, with a power of $91 \%$. Accordingly, $93 \%$ of the OSA subgroup had a positive SCR with significantly higher score $(8.7 \pm 2.2$ versus $7.3 \pm 1.9$ among habitual snorers; $\mathrm{p}=0.0001)$. Among the OSA subgroup, the BMI and pBMI were significantly lower compared with the habitual snoring subgroup ( $\mathrm{p}=0.031$ and 0.01 , respectively).

Among the SCR parameters oral breathing score, nasal hypotonia, saddle nose and tonsillar hypertrophy were significantly more commonly detected during clinical examination of preschool children in the OSA subgroup versus those in the habitual snoring subgroup (50.6\%, 34.6\%, 37.1\% and $73.4 \%$ versus $31 \%$, $18.2 \%, 14.5 \%$ and $45.5 \%$, respectively; $\mathrm{p}=0.011,0.021,0.002$ and $<0.0001$, respectively). 
FIGURE 1 Receiver operating characteristic analysis of the sleep clinical record for predicting obstructive sleep apnoea in preschool children larea under the curve $0.663,95 \% \mathrm{Cl} 0.606-0.717$; $\mathrm{p}=0.0001)$.

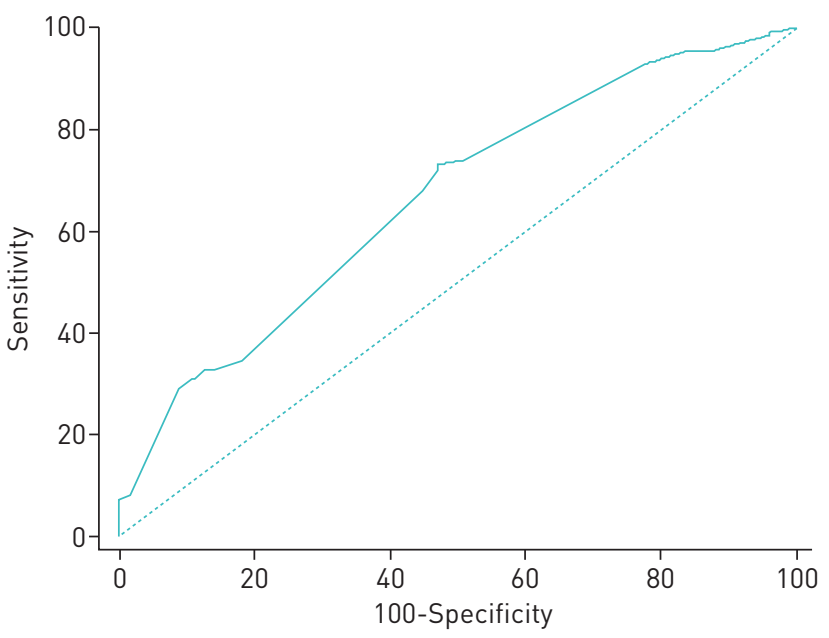

Univariate logistic regression analysis showed that tonsillar hypertrophy is the only significantly detected risk factor for development of OSA among school-age children with a hazard ratio of 2.3 (95\% CI 1.1-4.9; $\mathrm{p}=0.032$ ). While univariate logistic regression analysis among preschool children showed that a positive SCR is considered a risk factor for development of OSA with hazard ratio of 3.61 (95\% CI 1.61-8.11; $\mathrm{p}=0.002$ ) (table 2). Moreover, different parameters of the SCR showed significant hazard ratios to predict OSA, namely, in order of risk: saddle nose (hazard ratio of 3.42; $\mathrm{p}=0.002$ ), nasal hypotonia (hazard ratio of 2.34; $\mathrm{p}=0.024$ ), tonsillar grading score (hazard ratio of $1.82 ; \mathrm{p}<0.0001$ ) and oral breathing score (hazard

\section{TABLE 2 Univariate logistic regression analyses of different variables among preschool} children

\begin{tabular}{|c|c|c|c|c|}
\hline & B & SE & p-value & Hazard ratio $(95 \% \mathrm{CI})$ \\
\hline SCR (ADHD not included) & 1.284 & 0.412 & 0.002 & $3.61(1.61-8.11)$ \\
\hline Body mass index & -0.021 & 0.288 & 0.474 & $0.81(0.46-1.43)$ \\
\hline Body mass index percentile & -0.370 & 0.134 & 0.006 & $0.69(0.53-0.9)$ \\
\hline Nasal septal deviation & -0.261 & 0.347 & 0.453 & $0.77(0.39-1.52)$ \\
\hline Habitual obstruction & 0.658 & 0.364 & 0.071 & $1.93(0.95-3.94)$ \\
\hline Nasal mucosal hyperaemia & -0.524 & 0.295 & 0.076 & $0.59(0.33-1.06)$ \\
\hline Nasal secretions & 0.368 & 0.300 & 0.219 & $1.44(0.8-2.6)$ \\
\hline Turbinate hypertrophy & 0.483 & 0.260 & 0.063 & $1.62(0.97-2.7)$ \\
\hline Nasal obstruction & 0.269 & 0.183 & 0.140 & $1.31(0.92-1.87)$ \\
\hline Oral breathing score & 0.402 & 0.160 & 0.012 & $1.49(1.09-2.05)$ \\
\hline Nasal hypotonia & 0.851 & 0.376 & 0.024 & $2.34(1.12-4.9)$ \\
\hline Saddle nose & 1.229 & 0.406 & 0.002 & $3.42(1.54-7.58)$ \\
\hline Tonsillar grading score & 0.599 & 0.154 & $<0.0001$ & $1.82(1.35-2.46)$ \\
\hline Friedman Score & 0.037 & 0.193 & 0.848 & $1.04(0.71-1.52)$ \\
\hline Arched palate score & 0.281 & 0.434 & 0.518 & $1.32(0.57-3.1)$ \\
\hline $\begin{array}{l}\text { Angle class (normal versus } \\
\text { retrognathia versus prognathia) }\end{array}$ & 0.392 & 0.293 & 0.181 & $1.48(0.83-2.63)$ \\
\hline Angle score & 0.349 & 0.219 & 0.111 & $1.42(0.92-2.18)$ \\
\hline Open bite & -0.203 & 0.379 & 0.593 & $0.82(0.39-1.72)$ \\
\hline Deep bite & -0.01 & 0.315 & 0.975 & $0.99(0.53-1.84)$ \\
\hline Cross bite & -0.141 & 0.347 & 0.684 & $0.87(0.44-1.71)$ \\
\hline Overjet & 0.739 & 1.063 & 0.488 & $2.09(0.26-16.8)$ \\
\hline Skeletal occlusion & 0.085 & 0.169 & 0.618 & $1.09(0.78-1.52)$ \\
\hline Brouillette score & 0.357 & 0.384 & 0.353 & $1.43(0.67-3.03)$ \\
\hline \multicolumn{5}{|l|}{ Symptoms } \\
\hline Apnoea & 1.029 & 0.364 & 0.005 & $2.8(1.37-5.71)$ \\
\hline Snoring & 1.254 & 0.563 & 0.026 & $3.51(1.16-10.56)$ \\
\hline Restless sleep & 0.252 & 0.134 & 0.061 & $1.29(0.99-1.67)$ \\
\hline
\end{tabular}

SCR: sleep clinical record; ADHD: attention deficit hyperactivity disorder. Bold indicates statistical significance. 
ratio of 1.49; $\mathrm{p}=0.012$ ) as items of examination; and apnoea and snoring as items of symptomatology (hazard ratios of 2.8 and 3.51, p=0.005 and 0.026, respectively) (table 2). Furthermore, saddle nose, tonsillar grading score and oral breathing score as well as apnoea were statistically significant risk factors in the multivariate analysis ( $\mathrm{p}=0.003,0.003,0.014$, and 0.025 respectively; table 3 ).

\section{Discussion}

The current study presents the validity of the SCR, without the ADHD scale, in the evaluation of preschool children suspected to have OSA with a high hazard ratio. In our previous paper [8], we combined both clinical examination and history into the SCR and it was validated for differentiating children with OSA and habitual snoring with increasing probability $(89 \%)$ if the score was $\geqslant 6.5$. But we did not demonstrate the validity of the SCR in relation to age. In the current study, we demonstrated the validity of the SCR to identify preschool children at risk for OSA with a hazard ratio of 3.6. In addition, we demonstrated that the SCR, using the same cut-off point validated in our previous publication [8], is still valid even with the omission of ADHD rating scale, with a sensitivity of $74 \%$ and PPV of $86 \%$ to predict OSA indicating the validity of the SCR as an efficient screening tool in preschool children. Further, power analysis for the evaluated SCR was $91 \%$, which indicates a high chance of detecting a difference between OSA and habitual snoring in the preschool children [14]. In our previous study [8], no differences were identified in the percentage of children with a positive ADHD rating scale score between subjects with OSA and primary snorers. Although a significant improvement in diurnal neurobehavioral disorders has been widely demonstrated following adenotonsillectomy [15], the relationship between the severity of OSAS and cognitive deficits is usually weak [16]. In the present study, saddle nose, nasal hypotonia and oral breathing as well as tonsillar hypertrophy are considered as risk factors for development of OSA in preschool children group compared with only tonsillar hypertrophy among school-age children. To our knowledge, there is no specific validated questionnaire predicting OSA in young children $<6$ years of age. Interestingly, in preschool children the functional and structural aspects (saddle nose, oral breathing and lip hypotonia) associated with tonsillar hypertrophy are considered risk factors in those with OSA; while in school-age children remaining tonsillar hypertrophy and the consequences of oral breathing and nasal obstruction, such as nasal septum deviation and overjet, appear to be associated with the development of OSA. This suggests that identification of children with OSAS can occur early when you consider the importance of not only tonsillar hypertrophy and the presence of OSA, but also persistent oral breathing and loss of function of the nose.

PSG remains the gold standard for SDB diagnosis; however, this tool is still expensive and challenging especially in low resource areas $[17,18]$. Several questionnaires have been developed to identify children at risk of OSA [12, 19]. Recently, BERTRAN et al. [20] and KADMON et al. [21] demonstrated the validity of simple six- and eight-item paediatric sleep questionnaires, respectively, to identify children with OSA. However, all of these questionnaires have been mainly tested in school-age children.

In accordance with our results, KANG et al. [22] recently found that combining a number of anatomical findings (including adenoid/tonsillar size and obesity) and associated symptoms is effective for screening OSA in children aged from 2 to 18 years old.

\begin{tabular}{|c|c|c|c|c|}
\hline & B & SE & p-value & Hazard ratio $(95 \% \mathrm{Cl})$ \\
\hline Nasal septum deviation & -0.117 & 0.379 & 0.758 & $0.89(0.42-1.87)$ \\
\hline Oral breathing score & 0.471 & 0.191 & 0.014 & $1.6(1.1-2.33)$ \\
\hline Nasal obstruction & 0.157 & 0.227 & 0.49 & $1.17(0.75-1.83)$ \\
\hline Saddle nose & 1.545 & 0.525 & 0.003 & $4.69(1.67-13.13)$ \\
\hline Tonsillar grading score & 0.545 & 0.185 & 0.003 & $1.72(1.2-2.48)$ \\
\hline Friedman score & 0.148 & 0.249 & 0.552 & $1.16(0.71-1.89)$ \\
\hline Angle score & 0.394 & 0.281 & 0.162 & $1.48(0.85-2.57)$ \\
\hline Skeletal occlusion score & 0.211 & 0.203 & 0.299 & $1.24(0.83-1.84)$ \\
\hline Apnoea & 1.011 & 0.450 & 0.025 & $2.75(1.14-6.63)$ \\
\hline Snoring & 0.439 & 0.675 & 0.516 & $1.55(0.41-5.82)$ \\
\hline Constant & -1.76 & 0.781 & 0.024 & 0.17 \\
\hline
\end{tabular}


Tonsillar hypertrophy is an important risk factor for developing OSA in all age groups, and is the sole risk factor in older children with hazard ratio of 2.3. KADITIs et al. [23] hypothesised that tonsillar tissue in young children with SDB occupies a larger proportion of the oropharyngeal space compared with young subjects without SDB and with older age groups. This proportion may decrease in subjects without SDB, but remains unchanged in children with snoring resulting in persistent restriction of the upper airway lumen [23].

KANG et al. [24] and DAYYAT et al. [25] found that tonsils grade was positively correlated with obstructive AHI over a wide range of ages between 1 and 18 years old. Whereas in the current study, the presence of skeletal malocclusion, in the form of angle malocclusion (retrognathia/prognathia) and overjet, was significantly more common among school-age children with OSA compared with the preschool group. Craniofacial anatomical factors are believed to play a significant role in OSAS, together with upper airway compliance and muscle function mechanisms [26, 27]. DENG et al. [28] found that a retrusive mandible and deficient chin are commonly detected in children aged 6-12 years presenting with OSA when compared with healthy controls. Similarly in earlier studies, HANs et al. [29] and OzDEMIR et al. [30] found that snoring children have more deficient mandibles and more retrusive mandibles compared with control groups.

The current study illustrates others factors that show significant hazard ratios, in both univariate and multivariate analysis, in predicting OSA in children $<6$ years of age; in particular, those related to nasal hypotonia and oral breathing as well as saddle nose have significantly higher hazard ratios than tonsillar hypertrophy (tables 2 and 3). This could be explained by multiple factors. First, abnormal nasal resistance developed early in life leads to mouth breathing as well as associated oral-facial hypotonia and abnormal muscle tone [31, 32]. Secondly, infants presenting with abnormal oral-facial features, particularly a high and narrow hard palate, usually have associated oral-facial hypotonia, which has been shown to lead to persistence of abnormal tongue position and presence of mouth breathing detected by PSG in a follow-up study of preterm and full-term infants at 36 months of age [33]. This point concurs with our results as a high arched palate was significantly more prevalent in younger children (90\%) compared with the older group (78\%). Guilleminault et al. [34] found that persistence of mouth breathing during sleep, related to myofacial hypotonia, led to the recurrence of SDB after surgical management. On the same topic, VILLA et al. [35] considered oropharyngeal exercises as a complementary therapy to adenotonsillectomy in childhood OSA. In the current study, the saddle nose deformity in younger age group could be additional anatomical factor increasing nasal resistance and predisposing to oral breathing.

It's worth mentioning that in preschool children, significantly lower BMI was detected among those with OSA, which could be explained on the basis of increased inflammation associated with this disorder [36] affecting growth as demonstrated by NACHALON et al. [37] who reported improved somatic growth in young children aged 6-36 months of age after adenotonsillectomy. By contrast, obesity in the school-age group was significantly more prevalent $(40.6 \%)$ compared with the preschool group. Obesity is well known as a risk factor for OSA in school-age children and adolescents and frequently coexists $[38,39]$. It is worth noting that both OSA and obesity have been assumed to interact and promote one another [40].

\section{Conclusion}

The SCR is a valid screening tool in preschool children suspected to have OSA, even without the ADHD rating scale, and it is easy to apply. Oral breathing, nasal hypotonia, saddle nose and tonsillar hypertrophy are more commonly encountered in preschool children with OSA and constitute strong risk factors in this group; while obesity, malocclusions and overjet are more prevalent in school-age children with OSA. These clinical findings could be considered possible consequences of orofacial myofunctional dysfunction, related to SDB, in early life. Although tonsillar hypertrophy represented the single important risk factor for the development of OSA in school- age children, all the clinical parameters of children with SDB should be considered for integrated and resolutive treatment.

\section{References}

Tauman R, Gozal D. Obstructive sleep apnea syndrome in children. Expert Rev Respir Med 2011; 5: 425-440. Marcus CL, Brooks LJ, Draper KA, et al. Diagnosis and management of childhood obstructive sleep apnea syndrome. Pediatrics 2012; 130: 576-584.

3 Leiberman A, Stiller-Timor L, Tarasiuk A, et al. The effect of adenotonsillectomy on children suffering from obstructive sleep apnea syndrome (OSAS): the Negev perspective. Int J Pediatr Otorhinolaryngol 2006; 70: $1675-1682$.

4 Almeida LA, Anselmo-Lima WT, Valera FC. OSAS in children: where are we? Braz J Otorhinolaryngol 2011; 77: 273.

5 Hoban TF. Obstructive sleep apnea in children. Curr Treat Options Neurol 2005; 7: 353-361.

6 Powell S, Kubba H, O’Brien C, et al. Paediatric obstructive sleep apnoea. BMJ 2010; 340: c1918.

7 Berry RB, Budhiraja R, Gottlieb DJ, et al. Rules for scoring respiratory events in sleep: update of the 2007 AASM Manual for the Scoring of Sleep and Associated Events. Deliberations of the Sleep Apnea Definitions Task Force of the American Academy of Sleep Medicine. J Clin Sleep Med 2012; 8: 597-619. 
8 Villa MP, Paolino MC, Castaldo R, et al. Sleep clinical record: an aid to rapid and accurate diagnosis of paediatric sleep disordered breathing. Eur Respir J 2013; 41: 1355-1361.

9 Iber C, Ancoli-Israel S, Chesson AL, et al. The AASM Manual for the Scoring of Sleep and Associated Events: Rules, Terminology, and Technical Specifications. 1st Edn. Westchester, American Academy of Sleep Medicine, 2007.

10 Friedman M, Ibrahim H, Joseph NJ. Staging of obstructive sleep apnea/hypopnea syndrome: a guide to appropriate treatment. Laryngoscope 2004; 114: 454-459.

11 Liistro G, Rombaux P, Belge C, et al. High Mallampati score and nasal obstruction are associated risk factors for obstructive sleep apnoea. Eur Respir J 2003; 21: 248-252.

12 Brouilette R, Hanson D, David R, et al. A diagnostic approach to suspected obstructive sleep apnea in children. J Pediatr 1984; 105: 10-14.

13 Marzocchi GM CC. Una scala di facile uso per la rilevazione dei comportamenti problematici dei bambini con Deficit di Attenzione e Iperattività [A scale to indentify behavioural problems in ADHD children]. Psicologia Clinica dello Sviluppo 2000; 4: 43-64.

14 Suresh K, Chandrashekara S. Sample size estimation and power analysis for clinical research studies. J Hum Reprod Sci 2012; 5: 7-13.

15 Chervin RD, Ruzicka DL, Archbold KH, et al. Snoring predicts hyperactivity four years later. Sleep 2005; 28: 885-890.

16 Miano S, Paolino MC, Urbano A, et al. Neurocognitive assessment and sleep analysis in children with sleep-disordered breathing. Clin Neurophysiol 2011; 122: 311-319.

17 Gozal D, Kheirandish-Gozal L. New approaches to the diagnosis of sleep-disordered breathing in children. Sleep Med 2010; 11: 708-713.

18 Nixon GM, Brouillette RT. Diagnostic techniques for obstructive sleep apnoea: is polysomnography necessary? Paediatr Respir Rev 2002; 3: 18-24.

19 Brietzke SE, Katz ES, Roberson DW. Can history and physical examination reliably diagnose pediatric obstructive sleep apnea/hypopnea syndrome? A systematic review of the literature. Otolaryngol Head Neck Surg 2004; 131 827-832.

20 Bertran K, Mesa T, Rosso K, et al. Diagnostic accuracy of the Spanish version of the Pediatric Sleep Questionnaire for screening of obstructive sleep apnea in habitually snoring children. Sleep Med 2015; 16: 631-636.

21 Kadmon G, Chung SA, Shapiro CM. I'M SLEEPY: a short pediatric sleep apnea questionnaire. Int J Pediatr Otorhinolaryngol 2014; 78: 2116-2120.

22 Kang KT, Weng WC, Lee $\mathrm{CH}$, et al. Detection of pediatric obstructive sleep apnea syndrome: history or anatomical findings?. Sleep Med 2015; 16: 617-624

23 Kaditis AG, Lianou L, Hatzinikolaou S, et al. Tonsillar size in 2- to 14-year-old children with and without snoring. Pediatr Pulmonol 2009; 44: 1216-1222.

24 Kang KT, Chou CH, Weng WC, et al. Associations between adenotonsillar hypertrophy, age, and obesity in children with obstructive sleep apnea. PLoS One 2013; 8: e78666.

25 Dayyat E, Kheirandish-Gozal L, Sans Capdevila O, et al. Obstructive sleep apnea in children: relative contributions of body mass index and adenotonsillar hypertrophy. Chest 2009; 136: 137-144.

26 Ekici M, Ekici A, Keles H, et al. Risk factors and correlates of snoring and observed apnea. Sleep Med 2008; 9: 290-296.

27 Tsuda H, Fastlicht S, Almeida FR, et al. The correlation between craniofacial morphology and sleep-disordered breathing in children in an undergraduate orthodontic clinic. Sleep Breath 2011; 15: 163-171.

28 Deng J, Gao X. A case-control study of craniofacial features of children with obstructed sleep apnea. Sleep Breath 2012; 16: 1219-1227.

29 Hans MG, Nelson S, Pracharktam N, et al. Subgrouping persons with snoring and/or apnea by using anthropometric and cephalometric measures. Sleep Breath 2001; 5: 79-91.

30 Ozdemir H, Altin R, Sogut A, et al. Craniofacial differences according to AHI scores of children with obstructive sleep apnoea syndrome: cephalometric study in 39 patients. Pediatr Radiol 2004; 34: 393-399.

31 Miller AJ, Vargervik K, Chierici G. Experimentally induced neuromuscular changes during and after nasal airway obstruction. Am J Orthod 1984; 85: 385-392.

32 Vargervik K, Miller AJ, Chierici G, et al. Morphologic response to changes in neuromuscular patterns experimentally induced by altered modes of respiration. Am J Orthod 1984; 85: 115-124.

33 Huang YS, Guilleminault C. Pediatric obstructive sleep apnea and the critical role of oral-facial growth: evidences. Front Neurol 2012; 3: 184

34 Guilleminault C, Huang YS, Lin C, et al. Pediatric OSA, myo-facial re-education and facial growth. J Sleep Res 2012; 21: Suppl. 1, 70

35 Villa MP, Brasili L, Ferretti A, et al. Oropharyngeal exercises to reduce symptoms of OSA after AT. Sleep Breath 2015; 19: 281-289.

36 Tan HL, Gozal D, Wang Y, et al. Alterations in circulating T-cell lymphocyte populations in children with obstructive sleep apnea. Sleep 2013; 36: 913-922.

37 Nachalon Y, Lowenthal N, Greenberg-Dotan S, et al. Inflammation and growth in young children with obstructive sleep apnea syndrome before and after adenotonsillectomy. Mediators Inflamm 2014; 2014: 146893.

38 Verhulst SL, Schrauwen N, Haentjens D, et al. Sleep-disordered breathing in overweight and obese children and adolescents: prevalence, characteristics and the role of fat distribution. Arch Dis Child 2007; 92: 205-208.

39 Wing YK, Hui SH, Pak WM, et al. A controlled study of sleep related disordered breathing in obese children. Arch Dis Child 2003; 88: 1043-1047.

40 Bhattacharjee R, Kim J, Kheirandish-Gozal L, et al. Obesity and obstructive sleep apnea syndrome in children: a tale of inflammatory cascades. Pediatr Pulmonol 2011; 46: 313-323. 